10,12

\title{
Особенности тангенциальных мод в спектрах комбинационного рассеяния света одностенных полупроводниковых углеродных нанотрубок большого диаметра
}

\author{
() Д.И. Левшов ${ }^{1}$, Ю.С. Слабодян ${ }^{1}$, А.А. Тонких ${ }^{1}$, Т. Мiche/ ${ }^{2}$, С.Б. Рошаль ${ }^{1}$, Ю.И. Юзюк ${ }^{1}$ \\ ${ }^{1}$ Южный фредеральный университет, \\ Ростов-на-Дону, Россия \\ ${ }^{2}$ Laboratoire Charles Coulomb UMR 5221, Université de Montpellier, CNRS, \\ Montpellier, France \\ E-mail: dmitry.levshov@gmail.com
}

(Поступила в Редакцию 13 апреля 2016 г.

В окончательной редакции 23 августа 2016 г.)

\begin{abstract}
Методом резонансной спектроскопии комбинационного рассеяния света в широком диапазоне длин волн лазерного возбуждения исследована динамика решетки и оптические свойства индивидуальных полупроводниковых одностенных углеродных нанотрубок с диаметрами более $2.0 \mathrm{~nm}$. Индивидуальные подвешенные нанотрубки синтезированы методом химического осаждения из парогазовой фазы. Индексы хиральности $(n, m)$ нанотрубок определялись методом электронной дифракции. Обнаружена зависимость отношения абсолютных интенсивностей поперечной и продольной оптических тангенциальных мод как от энергии возбуждения, так и от структурных параметров нанотрубок (диаметра и хирального угла). Наблюдаемые зависимости отличаются от спектральных данных, полученных ранее для индивидуальных полупроводниковых структурно идентифицированных нанотрубок с диаметрами менее $1.1 \mathrm{~nm}$.
\end{abstract}

Работа выполнена за счет гранта Российского научного фонда (проект № 15-12-10004).

DOI: 10.21883/FTT.2017.03.44173.137

\section{1. Введение}

Спектроскопия комбинационного рассеяния света (КРС) является одним из наиболее популярных неразрушающих методов анализа атомной структуры одностенных углеродных нанотрубок (ОУНТ) [1]. Типичная процедура определения индексов хиральности $(n, m)$ нанотрубки заключается в вычислении диаметра $d$ ОУНТ по частоте радиально-дыхательной моды (RBM) с последующим сопоставлением теоретических и экспериментальных энергий электронных переходов $E_{i i}$ по графику Катаура [2-7]. Следует отметить, что успешность применения этой методики напрямую зависит от окружения, в котором находится нанотрубка [8]. Взаимодействие нанотрубки с окружением способно изменять как частоты радиальных мод, так и энергии электронных переходов в ОУНТ [1,9], что в конечном итоге может привести к неоднозначному определению атомной структуры.

Для исключения ошибок при индексации углеродных нанотрубок (УНТ) очень важно проводить анализ дополнительных КРС-активных мод, таких, например, как продольные $\left(\mathrm{LO}\right.$ или $G^{+}$) и поперечные ( $\mathrm{TO}$ или $G^{-}$) оптические (тангенциальные) моды. Ранее было установлено, что число этих мод в спектре КРС, их форма и частота напрямую зависят от типа проводимости и диаметров ОУНТ [2,10,11]. В частности, авторы [12] изучили тангенциальные моды четырнадцати различных суспензий полупроводниковых нанотрубок с малым диаметром $(d<1.1 \mathrm{~nm})$ и обнаружили, что частоты и ин- тенсивности тангенциальных мод зависят от хирального угла $\theta$ и диаметра $d$, а также предложили методику определения диаметров и индексов $(n, m)$ одностенных полупроводниковых нанотрубок малого диаметра на основе спектроскопических характеристик тангенциальных мод.

Целью настоящей работы является исследование особенностей тангенциальных мод индивидуальных подвешенных одностенных нанотрубок большого диаметра $(d>2.0 \mathrm{~nm})$, в частности изучение зависимости интенсивностей тангенциальных мод от структурных параметров и энергии лазерного возбуждения $E_{\text {laser }}$. Подобные зависимости в дальнейшем могут быть использованы для определения индексов хиральности $(n, m)$ ОУНТ. Работа структурирована следующим образом. В разделе 2 представлена методика получения образцов индивидуальных ОУНТ, а также описаны особенности экспериментов по спектроскопии КРС и электронной дифракции. В разделе 3 содержатся результаты измерений частот и интенсивностей тангенциальных мод полупроводниковых углеродных нанотрубок, обсуждается их зависимость от длины волны возбуждения, а также проводится сравнение с экспериментальными данными других работ. В Заключении дается обобщение выполненных исследований.

\section{2. Синтез и методика исследований}

Образцы индивидуальных углеродных нанотрубок были получены методом химического осаждения из па- 


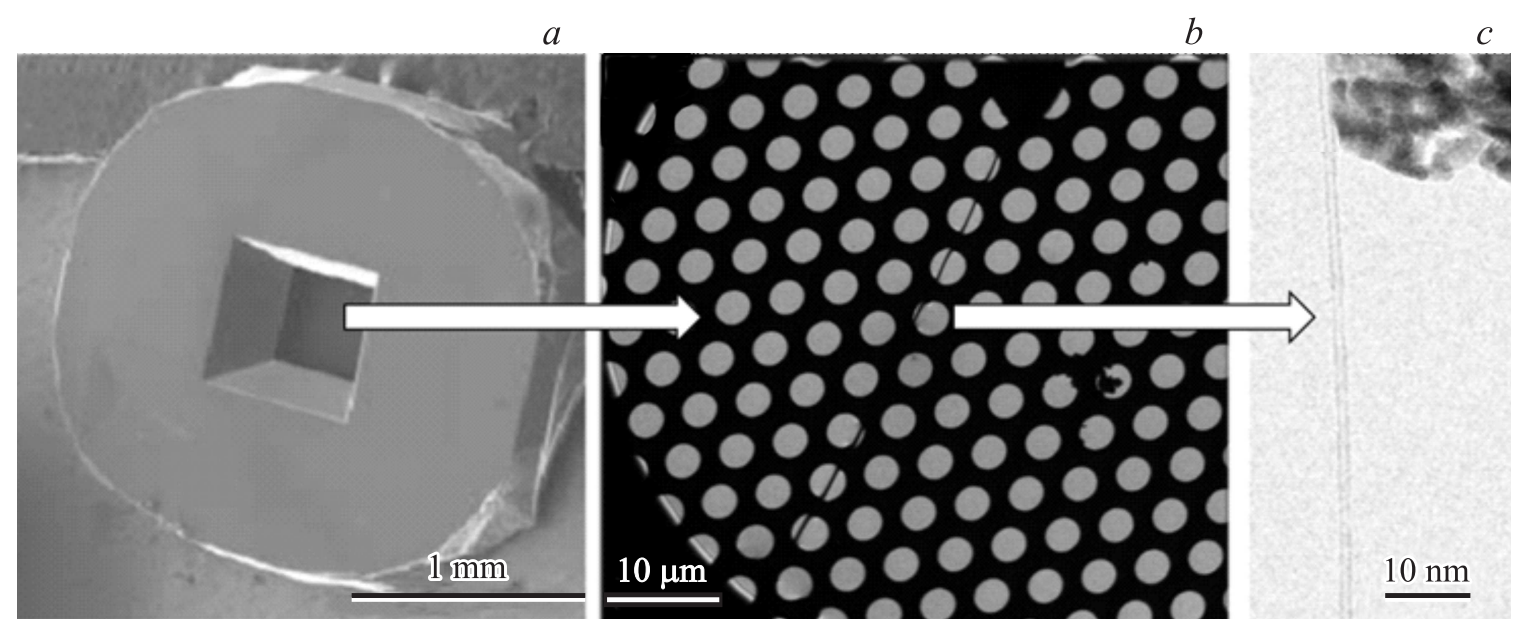

Рис. 1. Электронные изображения использованных при синтезе подложек $(a, b)$ и вид подвешенной нанотрубки $(c)$.

рогазовой фазы (CCVD), который ранее описывался в работах [3,4,13-18]. Использованные нами экспериментальные протоколы синтеза позволяют получать сверхдлинные нанотрубки (до $5 \mathrm{~cm}$ ), способные в процессе роста преодолевать траншеи (шириной в миллиметры) и барьеры (высотой $500 \mu \mathrm{m})$. В частности, в настоящей работе представлены нанотрубки, которые выращивались прямо на подложках для трансмиссионной микроскопии (перфорированные мембраны SPI, рис. 1,a). В образцах такого типа получаемые УНТ занимают несколько отверстий (рис. $1, b$ ). Типичный размер отверстий составляет $3 \mu \mathrm{m}$. На рис. $1, c$ показано полученное методом трансмиссионной электронной микроскопии изображение одной из УНТ на краю отверстия. Полученные электронные изображения показывают, что УНТ имеют высокую кристалличность и малую концентрацию дефектов.

Для однозначной интерпретации всех последующих экспериментов по спектроскопии КРС нами были определены индексы хиральности $(n, m)$ синтезированных индивидуальных нанотрубок методом электронной дифракции. Эксперименты по электронной дифракции и электронной микроскопии проводились на микроскопе FEI Titan Cubed Cs corrected 60-300 kV TEM при напряжении $80 \mathrm{kV}$. Электронные дифрактограммы записывались с использованием CCD-детектора (Gatan Ultrascan $2 K \times 2 K)$ с двумя типами камер: 285 и $460 \mathrm{~mm}$. Время накопления электронных дифрактограмм не превышало $10 \mathrm{~s}$. Особое внимание уделялось тому, чтобы электронный пучок не повреждал нанотрубки. Структурные параметры УНТ определялись согласно существующим в литературе процедурам, включая моделирование электронных дифрактограмм в рамках кинетической теории дифракции [19].

Эксперименты по спектроскопии резонансного КРС проводились на спектрометрах Jobin Yvon T64000, Renishaw InVia Reflex и Horiba IHR 320. Для получения максимальной спектральной информации спектры КРС углеродных нанотрубок измерялись в широком диапазоне длин волн лазерного возбуждения: 458, 476, 488, $514,532,568-633,647,676$ и 710-900 nm (дискретные лазеры $\mathrm{Ar}^{+}, \mathrm{Kr}^{+}, \mathrm{He}-\mathrm{Ne}$ и лазеры с перестраиваемой длиной волны Ti : sapphire и Dye laser). Рассеянный свет собирался с использованием $100 \times$ объектива $($ N.A. $=0.95)$ в конфигурации обратного рассеяния. Во всех измерениях поляризации падающего и рассеянного света были параллельны оси нанотрубки (\| -геометрия). Чтобы избежать нагревания нанотрубок, мощность лазерного излучения поддерживалась на уровне $50 \mu \mathrm{W}$. Параметры (частота, интенсивность, полуширина, интегральная площадь) основных КРС-активных линий были получены в результате моделирования спектров функциями Лоренца.

\section{3. Результаты и обсуждение}

В табл. 1 представлены структурные параметры и частоты основных КРС-активных мод одностенных полупроводниковых УНТ, исследованных в настоящей работе. Индексы $(n, m)$ нанотрубок находились напрямую из экспериментов по электронной дифракции. Семейство ОУНТ рассчитывается по формуле $v=(n-m) \bmod 3$ (ОУНТ является металлической, если $v=0$, и полупроводниковой при $v= \pm 1)$. Номер ветви (branch index) ОУНТ в графике Катаура задается числом $b=2 n+m$. Диаметр определяется по формуле $d=\left(\sqrt{3 a_{c-c}} \sqrt{n^{2}+n m+m^{2}} / \pi\right.$, где $a_{c-c}=0.142 \mathrm{~nm}-$ длина $\mathrm{C}-\mathrm{C}$-связи в структуре графена. Хиральный угол $\theta=\arccos \left((n+m / 2) / \sqrt{n^{2}+n m+m^{2}}\right) ; \omega_{\mathrm{RBM}}, \omega_{G^{+}}$и $\omega_{G^{-}}$ являются частотами радиально-дыхательной, продольной оптической тангенциальной $\left(\mathrm{LO}, G^{+}\right)$и поперечной оптической тангенциальной $\left(\mathrm{TO}, G^{-}\right)$колебательных мод ОУНТ соответственно.

В качестве примера для представления и обсуждения экспериментальных результатов выбраны две на- 
Таблица 1. Информация о структуре и динамике решетки исследованных в настоящей работе индивидуальных подвешенных полупроводниковых ОУНТ (экспериментальная погрешность в измерениях частот КРС мод равна $\pm 1 \mathrm{~cm}^{-1}$ )

\begin{tabular}{c|c|c|c|c|c|c|c|c|c}
\hline № $\Pi / \Pi$ & $(n, m)$ & $v$ & $b$ & $d, \mathrm{~nm}$ & $\theta, \mathrm{deg}$ & $1-\cos (6 \theta)$ & $\omega_{\mathrm{RBM}}, \mathrm{cm}^{-1}$ & $\omega_{G^{+}}, \mathrm{cm}^{-1}$ & $\omega_{G^{-}}, \mathrm{cm}^{-1}$ \\
\hline 1 & $(24,8)$ & 1 & 56 & 2.260 & 13.90 & 0.885 & 123 & 1591 & 1577 \\
2 & $(19,15)$ & 1 & 53 & 2.311 & 26.1 & 1.918 & 120 & 1590 & 1576 \\
3 & $(31,5)$ & 1 & 67 & 2.644 & 7.40 & 0.286 & 109.5 & 1591 & 1579.5
\end{tabular}

нотрубки из табл. 1: № 1 (24,8) и № 2 (19,15). Электронные дифрактограммы этих ОУНТ представлены на рис. 2, $a$ и $c$ соответственно. Рис. 2, $b$ демонстрирует зависимость спектров КРС нанотрубки $(24,8)$ от длины волны возбуждения (все спектры нормированы для лучшей иллюстрации относительной интенсивности TO- и LO-мод). Сверху вниз представлены спектры КРС, полученные при длинах волн возбуждения $458 \mathrm{~nm}(2.71 \mathrm{eV}), 488 \mathrm{~nm}(2.54 \mathrm{eV}), 514 \mathrm{~nm}$ $(2.41 \mathrm{eV}), 568 \mathrm{~nm}(2.18 \mathrm{eV}), 710 \mathrm{~nm}(1.75 \mathrm{eV})$ и $830 \mathrm{~nm}$ $(1.49 \mathrm{eV})$. Два последних спектра были выборочно взя- ты из диапазона $710-900 \mathrm{~nm}(1.38-1.75 \mathrm{eV})$, где с шагом $15 \mathrm{~nm}$ (7 nm вблизи резонанса) были выполнены измерения профилей резонансного возбуждения радиально-дыхательной моды и тангенциальных ТО- и LO-мод. Следует отметить, что при длинах волн возбуждающего излучения 488 и $830 \mathrm{~nm}$ некоторые из электронных переходов нанотрубки находятся в резонансе с падающим излучением (incident resonance). Это соответствует условию $E_{\text {laser }} \approx E_{i i}$, при котором в спектрах КРС видны как радиальная дыхательная мода $\left(\omega_{\mathrm{RBM}}=123 \mathrm{~cm}^{-1}\right.$, что эквивалентно $\left.\sim 15 \mathrm{meV}\right)$,
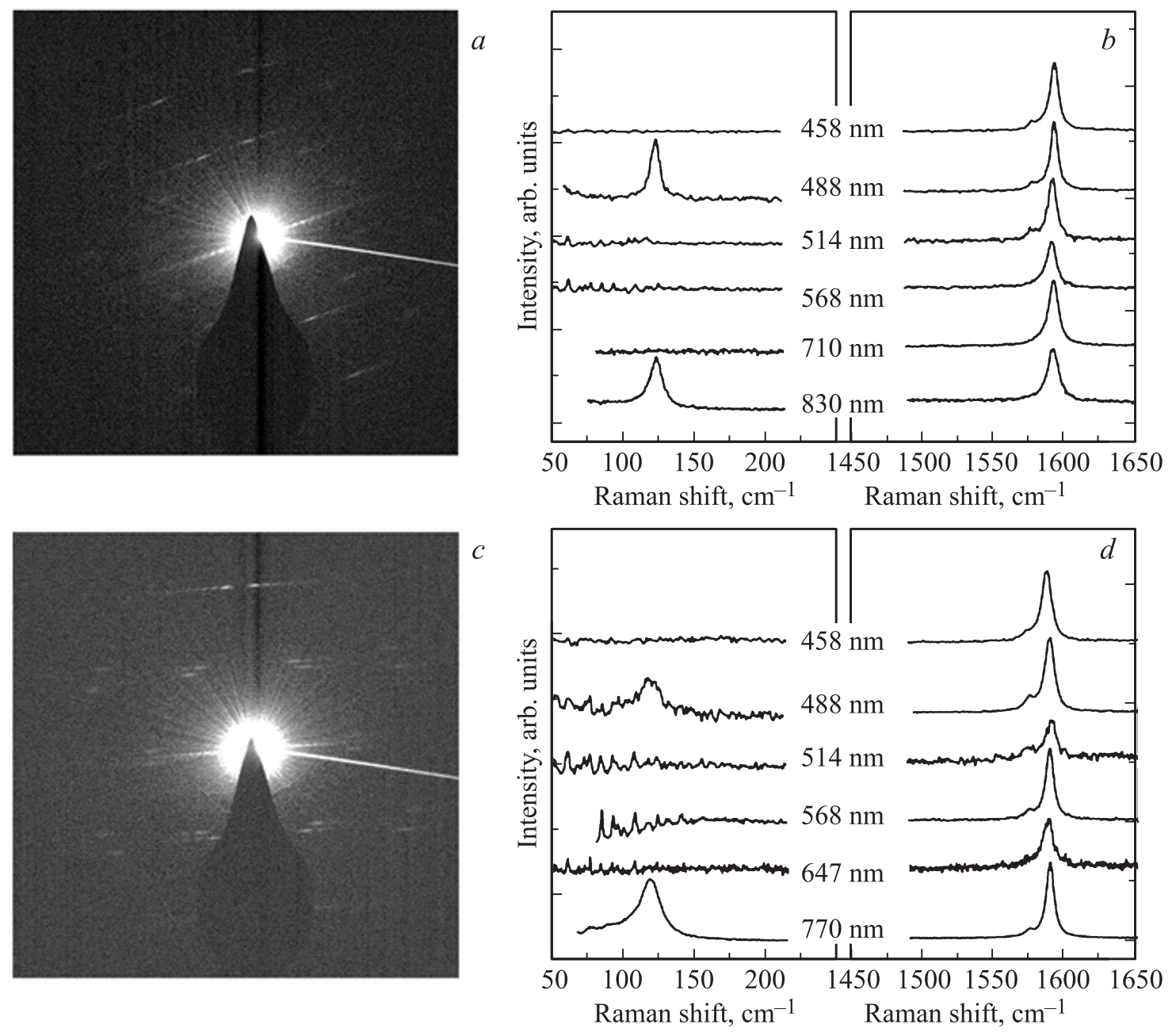

Рис. 2. Электронные дифрактограммы $(a, c)$ и спектры комбинационного рассеяния света $(b, d)$ одностенных полупроводниковых УНТ $(24,8)(a, b)$ и $(19,15) \quad(c, d)$. 
так и $G^{-}$полоса $\left(\omega_{G^{-}}=1577 \mathrm{~cm}^{-1}\right.$ и $\omega_{G^{+}}=1591 \mathrm{~cm}^{-1}$, что эквивалентно $\sim 195.5$ и $\sim 197 \mathrm{meV}$ соответственно). На всех других длинах волн выполняется условие резонанса с исходящим излучением (scattered/outgoing resonance) $E_{\text {laser }} \approx E_{i i}+E_{\text {phonon, при котором наблюда- }}$ ется только $G^{-}$-полоса, а радиально-дыхательной моды нет. Вне зависимости от длины волны возбуждения $G^{-}$-полоса имеет малую полуширину и состоит из двух компонент, что подтверждает полупроводниковый хиральный тип рассматриваемой ОУНТ [2]. Наконец, в спектрах не обнаружена $D$-полоса, что указывает на низкий уровень дефектов в исследуемой нанотрубке.

Аналогичные выводы о характере резонанса, типе проводимости и уровне дефектов можно сделать и для нанотрубки $(19,15)$ на основании спектров КРС, представленных на рис. 2, $d$. В этом случае радиальнодыхательная мода расположена на частоте $120 \mathrm{~cm}^{-1}$ $(\sim 15 \mathrm{meV})$, а две компоненты $G^{-}$-полосы имеют частоты 1576 и $1590 \mathrm{~cm}^{-1}$ ( 195 и $\sim 197 \mathrm{meV}$ соответственно). Небольшие осцилляции, наблюдаемые в низкочастотной области КРС-спектров, полученных при длинах волн возбуждения 514, 568 и $647 \mathrm{~nm}$, соответствуют КРС-активным модам воздуха. Данные осцилляции присутствуют в КРС-спектрах, измеряемых на воздухе вдали от нанотрубок.

Ранее авторы [12] измерили частоты тангенциальных мод небольших полупроводниковых нанотрубок $(d<1.1 \mathrm{~nm})$. Взяв за основу собственные измерения и данные [20] по частотам больших полупроводниковых ОУНТ $(d>2.0 \mathrm{~nm})$, авторы [12] установили выражение, связывающее частоту тангенциальных мод и диаметр одностенной полупроводниковой УНТ:

$$
\omega_{G}(d)=a_{0}+\frac{a_{1}}{d^{2}}+\frac{a_{2}}{d},
$$

где для LO-моды $a_{0}=1582 \mathrm{~cm}^{-1}, a_{1}=-14.9 \mathrm{~nm}^{2} / \mathrm{cm}$, $a_{2}=24.9 \mathrm{~nm} / \mathrm{cm}, \quad$ а для ТО-моды $a_{0}=1582 \mathrm{~cm}^{-1}$, $a_{1}=-27.5 \mathrm{~nm}^{2} / \mathrm{cm}, a_{2}=0 \mathrm{~nm} / \mathrm{cm}$.

Оценим по формуле (1) частоты тангенциальных мод для исследуемых УНТ. Для ОУНТ $(24,8)$ $\omega_{\text {ТО }}=1576.6 \mathrm{~cm}^{-1}, \quad \omega_{\mathrm{LO}}=1590.0 \mathrm{~cm}^{-1}$. Для ОУНТ $(19,15) \omega_{\mathrm{TO}}=1576.9 \mathrm{~cm}^{-1}, \omega_{\mathrm{LO}}=1590.0 \mathrm{~cm}^{-1}$. Таким образом, частоты LO- и TO-мод исследуемых нами ОУНТ находятся в хорошем согласии с данными работы [12].

Далее мы проанализировали зависимость интенсивности тангенциальных TO- и LO-мод ОУНT от энергии возбуждения $E_{\text {laser. }}$ Эти данные, в частности, показаны на рис. $3, a, b$ для ОУНТ $(24,8)$ и рис. $3, c, d$ для ОУНТ $(19,15)$. Подробная информация о спектральных параметрах основных КРС-активных линий, полученных в результате моделирования спектров функциями Лоренца, представлена в табл. 2 и 3. Из рис. $3, d$ ясно видно, что относительная интенсивность ТО-моды (в сравнении с интенсивностью LO-моды) нанотрубки $(19,15)$ при энергии возбуждения $2.54 \mathrm{eV}$ (панель 2) почти в 3 раза
Таблица 2. Зависимость от энергии возбуждения отношений абсолютных интенсивностей $I_{\mathrm{TO}} / I_{\mathrm{LO}}$ и интегральных интенсивностей $A_{\mathrm{TO}} / A_{\mathrm{LO}}$ для ОУНТ $(24,8)$

\begin{tabular}{c|c|c|c}
\hline $\begin{array}{c}\text { Длина волны } \\
\text { возбуждения, nm }\end{array}$ & $\begin{array}{c}\text { Энергия } \\
\text { возбуждения, } \mathrm{eV}\end{array}$ & $I_{\mathrm{TO}} / I_{\mathrm{LO}}$ & $A_{\mathrm{TO}} / A_{\mathrm{LO}}$ \\
\hline 458 & 2.71 & 0.0897 & 0.172 \\
488 & 2.54 & 0.0861 & 0.165 \\
514 & 2.41 & 0.0855 & 0.148 \\
568 & 2.18 & 0.0936 & 0.160 \\
710 & 1.75 & 0.0474 & 0.074 \\
725 & 1.71 & 0.0473 & 0.074 \\
740 & 1.68 & 0.0504 & 0.083 \\
755 & 1.65 & 0.0643 & 0.105 \\
762 & 1.63 & 0.0625 & 0.103 \\
770 & 1.61 & 0.0816 & 0.143 \\
777 & 1.60 & 0.0740 & 0.123 \\
785 & 1.58 & 0.0671 & 0.114 \\
805 & 1.54 & 0.0859 & 0.145 \\
830 & 1.49 & 0.0430 & 0.060
\end{tabular}

Таблица 3. Зависимость от энергии возбуждения отношений абсолютных интенсивностей $I_{\mathrm{TO}} / I_{\mathrm{LO}}$ и интегральных интенсивностей $A_{\mathrm{TO}} / A_{\mathrm{LO}}$ для ОУНТ $(19,5)$

\begin{tabular}{c|c|l|l}
\hline $\begin{array}{c}\text { Длина волны } \\
\text { возбуждения, } \mathrm{nm}\end{array}$ & $\begin{array}{c}\text { Энергия } \\
\text { возбуждения, } \mathrm{eV}\end{array}$ & $I_{\mathrm{TO}} / I_{\mathrm{LO}}$ & $A_{\mathrm{TO}} / A_{\mathrm{LO}}$ \\
\hline 458 & 2.71 & 0.08 & 0.10 \\
488 & 2.54 & 0.21 & 0.27 \\
514 & 2.41 & 0.25 & 0.29 \\
568 & 2.18 & 0.098 & 0.14 \\
710 & 1.75 & 0.05 & 0.08 \\
725 & 1.71 & 0.04 & 0.06 \\
740 & 1.68 & 0.05 & 0.08 \\
755 & 1.65 & 0.06 & 0.07 \\
762 & 1.63 & 0.07 & 0.10 \\
770 & 1.61 & 0.075 & 0.10 \\
777 & 1.60 & 0.08 & 0.10 \\
785 & 1.58 & 0.086 & 0.11 \\
805 & 1.54 & 0.09 & 0.19
\end{tabular}

больше, чем при энергии возбуждения $1.61 \mathrm{eV}$ (панель 4). Аналогично в нанотрубке $(24,8)$ (рис. $3, b)$ при энергии возбуждения $2.71 \mathrm{eV}$ (панель 2) относительная интенсивность ТО-моды в несколько раз выше, чем в инфракрасной области при $1.75 \mathrm{eV}$ (панель 1 ).

При этом абсолютное значение $I_{\mathrm{TO}} / I_{\mathrm{LO}}$ зависит от энергии возбуждаемого электронного перехода $E_{i i}$, a разница между максимальным и минимальным значениями $I_{\mathrm{TO}} / I_{\mathrm{LO}}$ зависит от индексов $(n, m)$ нанотрубки. Так, вблизи $E_{33}(\sim 1.60 \mathrm{eV}$, рис. $3, a, c)$ значение $I_{\mathrm{TO}} / I_{\mathrm{LO}}$ изменяется равномерно в пределах 0.04-0.08 как в ОУНТ $(24,8)$, так и в $(19,15)$. Этот результат в целом согласуется с данными работы [12], полученными для малых ОУНТ. Однако при энергиях возбуждения, близких к $E_{44}$ и $E_{55}$, значения $I_{\mathrm{TO}} / I_{\mathrm{LO}}$ 

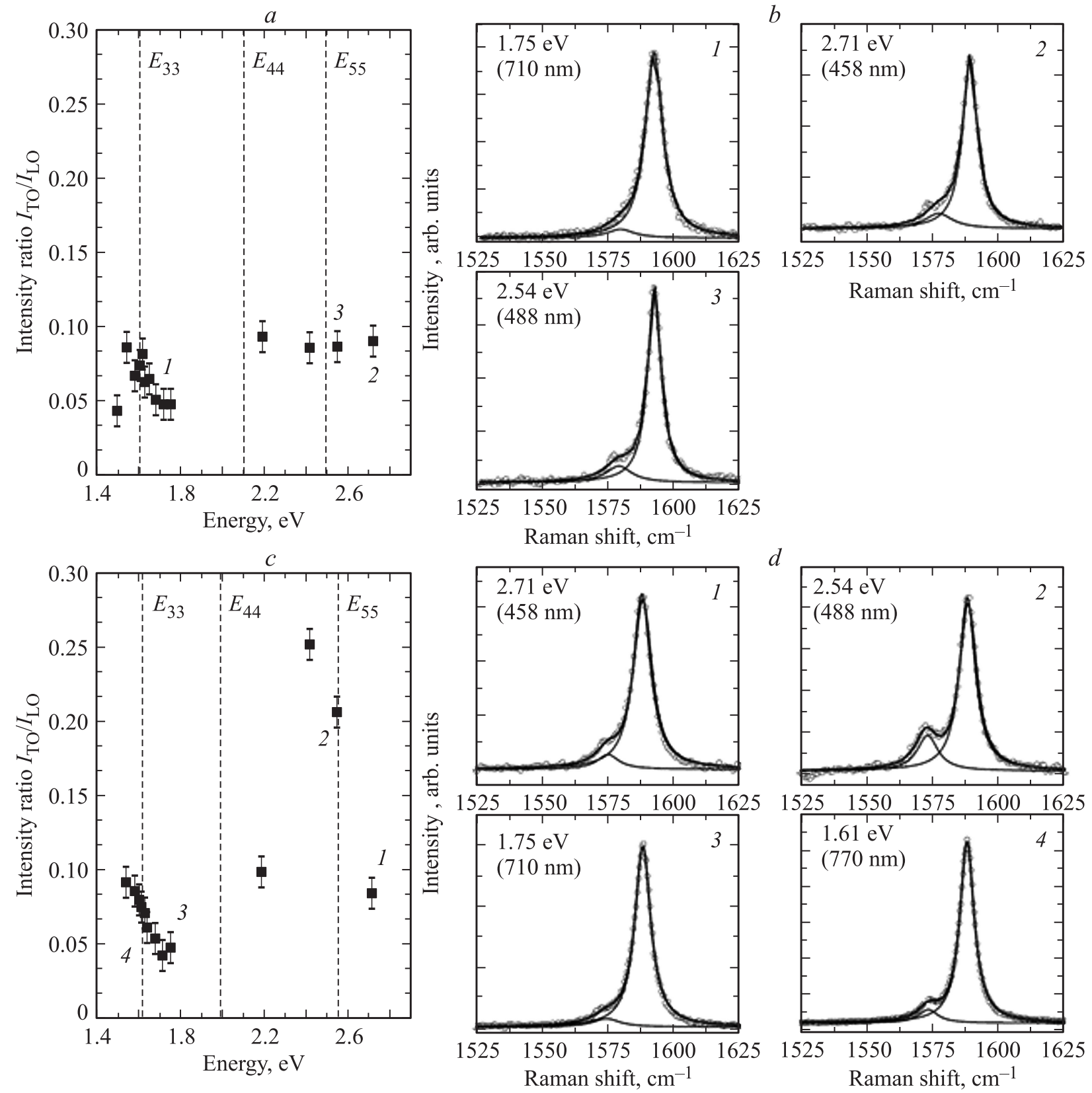

Рис. 3. Отношение абсолютных интенсивностей $I_{\mathrm{TO}} / I_{\mathrm{LO}}$ (или $I\left(G^{-}\right) / I\left(G^{+}\right)$как функция энергии возбуждения $E_{\text {laser }}$ для ОУНТ $(24,8)(a)$ и ОУНТ $(19,15)(c)$. Штриховыми линиями показаны оптические переходы с энергиями $E_{i i}$ в ОУНТ, рассчитанные согласно данным работы [21]. Область высокочастотных тангенциальных мод ОУНТ $(24,8)(b)$ и $(19,15)(d)$ при различных энергиях лазерного возбуждения. Для ОУНТ (24,8) панели 1-3 представляют энергии возбуждения $1.75 \mathrm{eV}(710 \mathrm{~nm}), 2.71 \mathrm{eV}$ $(458 \mathrm{~nm})$ и $254(488 \mathrm{~nm})$ соответственно. Для ОУНТ $(19,15)$ панели $1-4$ представляют энергии возбуждения $2.71 \mathrm{eV}(458 \mathrm{~nm})$, $2.54(488 \mathrm{~nm}), 1.75 \mathrm{eV}(710 \mathrm{~nm})$ и $1.61 \mathrm{eV}(770 \mathrm{~nm})$ соответственно. Ясно видно, что относительная интенсивность ТО-моды сильно зависит от энергии возбуждения.

составляют уже 0.09 и 0.25 для нанотрубок $(24,8)$ и $(19,15)$ соответственно. Подобная разница в значениях $I_{\mathrm{TO}} / I_{\mathrm{LO}}$ была отмечена авторами [12] при сравнении с данными работы [22] (случай больших ОУНТ), однако была объяснена повреждениями, возникающими в ОУНТ при химическом травлении в процессе подготовки образцов. В настоящей работе мы применили более совершенный протокол синтеза, исключающий любую химическую обработку нанотрубок. Следовательно, наблюдаемые зависимости интенсивностей тангенциальных мод являются характеристикой собственных свойств ОУНТ.

Далее мы проанализировали зависимость абсолютных интенсивностей тангенциальных мод $I_{\mathrm{TO}} / I_{\mathrm{LO}}$ от угла хиральности $\theta$ и диаметра $d$ полупроводниковых ОУНТ. На рис. 4, $a$ показано отношение $I_{\mathrm{TO}} / I_{\mathrm{LO}}$ как функция 

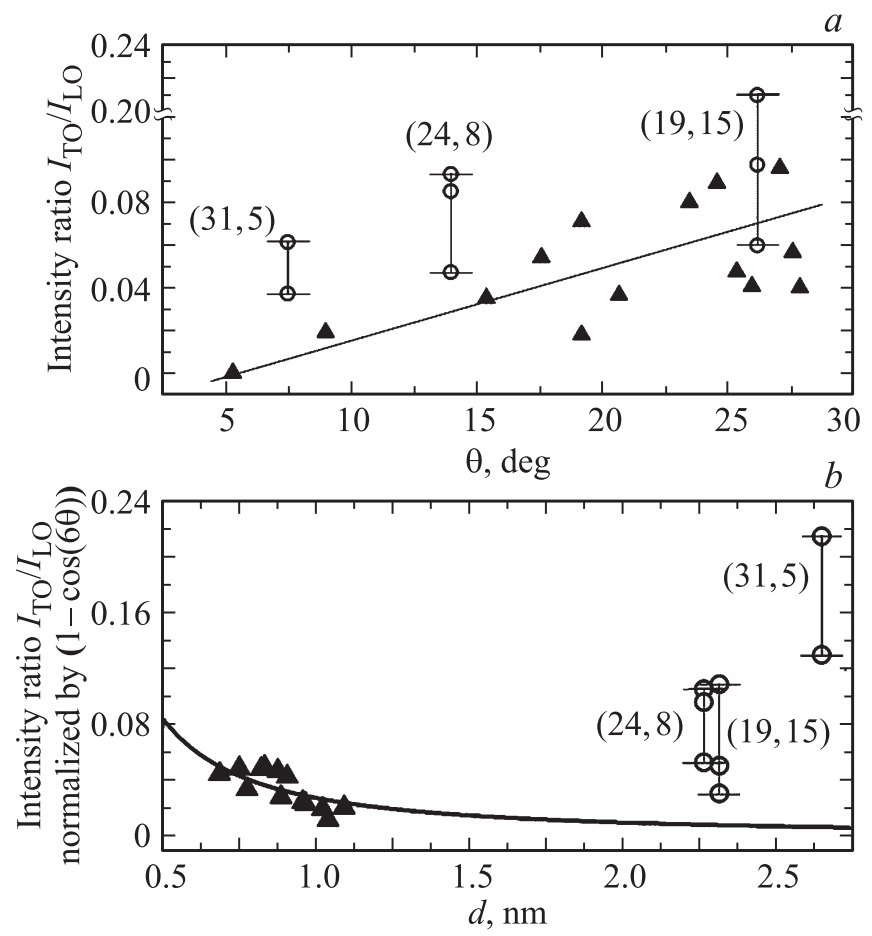

Рис. 4. Отношение интенсивностей ТO- и LO-мод $I_{\mathrm{TO}} / I_{\mathrm{LO}}$ как функция хирального угла $\theta(a)$ и диаметра $d(b)$ одностенных углеродных нанотрубок малого диаметра (треугольники, данные работы [12]) и ОУНТ большого диаметра (кружки, данные настоящей работы). Тонкая линия на части $a$ представлена для наглядности. Сплошная линия на части $b$ является результатом моделирования данных работы [12] по формуле (2).

хирального угла $\theta$ для наших ОУНТ (кружки) и нанотрубок из работы [12] (треугольники). Для нанотрубок $(24,8)$ и $(19,15)$ представлены три различных значения $I_{\mathrm{TO}} / I_{\mathrm{LO}}$ для переходов с энергиями $E_{33}, E_{44}$ и $E_{55}$, а для $(31,5)$ - два значения при $E_{44}$ и $E_{55}$ (энергии $E_{i i}$ определялись по эмпирической формуле (1) из работы [21]). Напомним, что в [12] авторы измерили спектры КРС только на одной длине волны для каждой отдельной ОУНТ (преимущественно возбуждались переходы с энергией $\left.E_{22}\right)$, соответственно на каждую нанотрубку приходилось только одно значение $I_{\mathrm{TO}} / I_{\mathrm{LO}}$. На основе этих данных авторы [12] обнаружили, что соотношение $I_{\mathrm{TO}} / I_{\mathrm{LO}}$ увеличивается с ростом хирального угла $\theta$, т.е. минимально для ОУНТ типа „зигзаг“" $\left(\theta=0^{\circ}\right)$ и максимально для ОУНТ типа „кресло“ $\left(\theta=30^{\circ}\right)$. Наблюдаемый разброс значений $I_{\mathrm{TO}} / I_{\mathrm{LO}}$ (см. отклонения треугольников от тонкой линии на рис. 4,a) авторы работы [12] связали с зависимостью $I_{\mathrm{TO}} / I_{\mathrm{LO}}$ от диаметра ОУНТ и предложили следующую формулу:

$$
\frac{I\left(G^{-}\right)}{I\left(G^{+}\right)}=\left(\frac{b_{1}}{d}+\frac{b_{2}}{d^{2}}\right)(1-\cos 6 \theta),
$$

где $b_{1}=0.014 \mathrm{~nm}, b_{2}=0.014 \mathrm{~nm}^{2}$.

На рис. 4, $b$ представлены результаты расчетов, сделанных по формуле (2) для наших нанотрубок (кружки) и данных работы [12] (треугольники). Из рисунка ясно видно, что между двумя наборами данных существует разногласие. Мы предполагаем, что это может быть связано с зависимостью $I_{\mathrm{TO}} / I_{\mathrm{LO}}$ от энергии возбуждения $E_{\text {laser }}$ и, как следствие, некорректностью формулы (2). Однако для однозначных выводов необходимо проведение дополнительных измерений на большем числе ОУНТ большого диаметра. Проведенные нами исследования указывают на то, что методики индексации полупроводниковых нанотрубок на основе анализа интенсивностей тангенциальных мод [12] следует применять с осторожностью (особенно в случае ОУНТ с диаметрами более $2.0 \mathrm{~nm})$.

\section{4. Заключение}

Исследованы зависимости интенсивностей тангенциальных мод от структурных параметров и энергии лазерного возбуждения в индивидуальных полупроводниковых одностенных углеродных нанотрубках, синтезированных методом осаждения из парогазовой фазы. Индексы хиральности $(n, m)$ нанотрубок были определены методом электронной дифракции. Эксперименты по спектроскопии резонансного КРС проводились в максимально широком диапазоне длин волн лазерного возбуждения $(458,476,488,514,532,568-633,647,676$ и $710-900 \mathrm{~nm})$.

Проведенные исследования ясно показали, что отношение абсолютных интенсивностей TO- и LO-мод $\left(I_{\mathrm{TO}} / I_{\mathrm{LO}}\right)$ зависит от энергии возбуждения $E_{\text {laser }}$ (или от энергии возбуждаемых в нанотрубке электронных переходов $\left.E_{i i}\right)$, а разница между максимальным и минимальным значениями $I_{\mathrm{TO}} / I_{\mathrm{LO}}$ зависит от индексов $(n, m)$ нанотрубки. Было также обнаружено, что установленные ранее [12] зависимости $I_{\mathrm{TO}} / I_{\mathrm{LO}}$ от диаметра $d$ и хирального угла $\theta$ не согласуются с данными, полученными на полупроводниковых ОУНТ с диаметрами более $2.0 \mathrm{~nm}$. В связи с этим предложенные в работе [12] методики индексации полупроводниковых нанотрубок на основе спектроскопических характеристик тангенциальных мод следует применять с осторожностью, особенно при изучении нанотрубок с диаметрами более $2.0 \mathrm{~nm}$.

\section{Список литературы}

[1] R. Saito, M. Hofmann, G. Dresselhaus, A. Jorio. Adv. Phys. 60, 413 (2011).

[2] M. Paillet, T. Michel, A. Zahab, D. Nakabayashi, V. Jourdain, R. Parret, J. Meyer, J.-L. Sauvajol. Phys. Status Solidi B 247, 2762 (2010).

[3] R. Parret, D. Levshov, T.X. Than, D. Nakabayashi, T. Michel, M. Paillet, R. Arenal, V.N. Popov, V. Jourdain, Y.I. Yuzyuk, A.A. Zahab, J.-L. Sauvajol. MRS Proc. 1407, mrsf11-1407aa07-01 (2012).

[4] D. Levshov, T. Michel, M. Paillet, X.T. Than, H.N. Tran, R. Arenal, A. Rahmani, M. Boutahir, A.-A. Zahab, J.-L. Sauvajol. MRS Proc. 1700, 69 (2014). 
[5] C. Fantini, A. Jorio, M. Souza, M. Strano, M. Dresselhaus, M. Pimenta. Phys. Rev. Lett. 93, 147406 (2004).

[6] H. Telg, J. Maultzsch, S. Reich, F. Hennrich, C. Thomsen. Phys. Rev. Lett. 93, 177401 (2004).

[7] J. Maultzsch, H. Telg, S. Reich, C. Thomsen. Phys. Rev. B 72, 205438 (2005).

[8] D.I. Levshov, Y.I. Yuzyuk, T. Michel, C. Voisin, L. Alvarez, S. Berger, P. Roussignol, J.-L. Sauvajol. J. Phys. Chem. C 114, 16210 (2010).

[9] P. Araujo, I. Maciel, P. Pesce, M. Pimenta, S. Doorn, H. Qian, A. Hartschuh, M. Steiner, L. Grigorian, K. Hata, A. Jorio. Phys. Rev. B 77, 241403 (2008).

[10] S. Piscanec, M. Lazzeri, J. Robertson, A. Ferrari, F. Mauri. Phys. Rev. B 75, 035427 (2007).

[11] V. Popov, P. Lambin. Phys. Rev. B 73, 085407 (2006).

[12] H. Telg, J.G. Duque, M. Staiger, X. Tu, F. Hennrich, M.M. Kappes, M. Zheng, J. Maultzsch, C. Thomsen, S.K. Doorn. ACS Nano 6, 904 (2012).

[13] T.X. Tinh, N. Van Chuc, V. Jourdain, M. Paillet, D.-Y. Kim, J.-L. Sauvajol, N.T.T. Tam, P.N. Minh. J. Exp. Nanosci. 6, 547 (2011).

[14] R. Arenal, P. Löthman, M. Picher, T. Than, M. Paillet, V. Jourdain. J. Phys. Chem. C 116, 14103 (2012).

[15] D. Levshov, T.X. Than, R. Arenal, V.N. Popov, R. Parret, M. Paillet, V. Jourdain, A.A. Zahab, T. Michel, Y.I. Yuzyuk, J.-L. Sauvajol. Nano Lett. 11, 4800 (2011).

[16] D. Levshov, T. Michel, T. Than, M. Paillet, R. Arenal, V. Jourdain, Y.I. Yuzyuk, J.-L. Sauvajol. J. Nanoelectron. Optoelectron. 8, 9 (2013).

[17] D.I. Levshov, M.V. Avramenko, X. Than, T. Michel, R. Arenal, M. Paillet, D.V. Rybkovskiy, A.V. Osadchy, S.B. Rochal, Y.I. Yuzyuk, J. Sauvajol. J. Nanophoton. 10, 012502 (2016).

[18] D.I. Levshov, T. Michel, R. Arenal, H.N. Tran, T.X.Than, M. Paillet, Y.I. Yuzyuk, J.-L. Sauvajol. J. Phys. Chem. C 119, 23196 (2015).

[19] C.S. Allen, C. Zhang, G. Burnell, A.P. Brown, J. Robertson, B.J. Hickey. Carbon 49, 4961 (2011).

[20] M. Paillet, T. Michel, J.C. Meyer, V.N. Popov, L. Henrard, S. Roth, J.L. Sauvajol. Phys. Rev. Lett. 96, 257401 (2006).

[21] K. Liu, J. Deslippe, F. Xiao, R.B. Capaz, X. Hong, S. Aloni, A. Zettl, W. Wang, X. Bai, S.G. Louie, E. Wang, F. Wang. Nature Nanotechnol. 7, 325 (2012).

[22] T. Michel, M. Paillet, D. Nakabayashi, M. Picher, V. Jourdain, J.C. Meyer, A.A. Zahab, J.-L. Sauvajol. Phys. Rev. B 80, 245416 (2009). 